Also available at http://amc.imfm.si

ISSN 1855-3966 (printed edn.), ISSN 1855-3974 (electronic edn.)

ARS MATHEMATICA CONTEMPORANEA 4 (2011) 153-164

\title{
Graphs with maximum degree 5 are acyclically 7-colorable*
}

\author{
Alexandr V. Kostochka ${ }^{\dagger}$ \\ Department of Mathematics, University of Illinois, Urbana, IL 61801, USA \\ Sobolev Institute of Mathematics, Novosibirsk, 630090, Russia \\ Christopher Stocker \\ Department of Mathematics, University of Illinois, Urbana, IL 61801, USA
}

Received 12 May 2010, accepted 21 January 2011, published online 24 March 2011

\begin{abstract}
An acyclic coloring is a proper coloring with the additional property that the union of any two color classes induces a forest. We show that every graph with maximum degree at most 5 has an acyclic 7 -coloring. We also show that every graph with maximum degree at most $r$ has an acyclic $\left(1+\left\lfloor\frac{(r+1)^{2}}{4}\right\rfloor\right)$-coloring.
\end{abstract}

Keywords: Acyclic coloring, maximum degree.

Math. Subj. Class.: 05C15, 05C35

\section{Introduction}

A proper coloring of the vertices of a graph $G=(V, E)$ is an assignment of colors to the vertices of the graph such that no two adjacent vertices receive the same color. A proper coloring of a graph $G$ is acyclic if the union of any two color classes induces a forest. The acyclic chromatic number, $a(G)$, is the smallest integer $k$ such that $G$ is acyclically $k$-colorable. The notion of acyclic coloring was introduced in 1973 by Grünbaum [8] and turned out to be interesting and closely connected to a number of other notions in graph coloring. Several researchers felt the beauty of the subject and started working on problems and conjectures posed by Grünbaum. Michael Albertson was among the enthusiasts and wrote in total four papers on the topic [1,2,3,4].

\footnotetext{
* Dedicated to the memory of Michael Albertson

${ }^{\dagger}$ Supported in part by NSF grants DMS-0650784 and DMS-0965587 and grant 09-01-00244-a of the Russian Foundation for Basic Research.

E-mail addresses: kostochk@math.uiuc.edu (Alexandr V. Kostochka), stocker2@illinois.edu (Christopher Stocker)
} 
In particular, Grünbaum studied $a(r)$ - the maximum value of the acyclic chromatic number over all graphs $G$ with maximum degree at most $r$. He conjectured that for every $r, a(r)=r+1$ and proved that his conjecture holds for $r \leq 3$. In 1979, Burstein [6] proved the conjecture for $r=4$. This result was proved independently by Kostochka [10]. It was also proved in [10] that for every $k \geq 3$, the problem of deciding whether a graph is acyclically $k$-colorable is NP-complete. It turned out that for large $r$, Grünbaum's conjecture is incorrect in a strong sense. Albertson and Berman mentioned in [1] that Erdôs proved that $a(r)=\Omega\left(r^{4 / 3-\epsilon}\right)$ and conjectured that $a(r)=o\left(r^{2}\right)$. Alon, McDiarmid and Reed [5] sharpened Erdős' lower bound to $a(r) \geq c r^{4 / 3} /(\log r)^{1 / 3}$ and proved that

$$
a(r) \leq 50 r^{4 / 3}
$$

This established almost the order of the magnitude of $a(r)$ for large $r$. Recently, the problem of estimating $a(r)$ for small $r$ was considered again.

Fertin and Raspaud [7] showed among other results that $a(5) \leq 9$ and gave a linear-time algorithm for acyclic 9-coloring of any graph with maximum degree 5 . Furthermore, for every fixed $r \geq 3$, they gave a fast algorithm that uses at most $r(r-1) / 2$ colors for acyclic coloring of any graph with maximum degree $r$. Of course, for large $r$ this is much worse than the upper bound (1.1), but for $r<1000$, it is better. Hocquard and Montassier [9] showed that every 5 -connected graph $G$ with $\Delta(G)=5$ has an acyclic 8-coloring. Kothapalli, Varagani, Venkaiah, and Yadav [12] showed that $a(5) \leq 8$. Kothapalli, Satish, and Venkaiah [11] proved that every graph with maximum degree $r$ is acyclically colorable with at most $1+r(3 r+4) / 8$ colors. This is better than the bound $r(r-1) / 2$ in [7] for $r \geq 8$. The main result of this paper is

Theorem 1.1. Every graph with maximum degree 5 has an acyclic 7-coloring, i.e., a $(5) \leq$ 7.

We do not know whether $a(5)$ is 7 or 6 , and do not have a strong opinion about it.

Our proof is different from that in [7, 9, 12] and heavily uses the ideas of Burstein [6]: he started from an uncolored graph $G$ with maximum degree 4 and colored step by step more and more vertices (with some recolorings) so that each of partial acyclic 5-colorings of $G$ had additional good properties that enabled him to extend the coloring further. The proof yields a linear-time algorithm for acyclic coloring with at most 7 colors of any graph with maximum degree 5 . Using this approach we also show that for every fixed $r \geq 6$, there exists a linear-time algorithm giving an acyclic coloring of any graph with maximum degree $r$ with at most $1+\left\lfloor\frac{(r+1)^{2}}{4}\right\rfloor$ colors. This is better than the bounds in [7] and [11] cited above for every $r \geq 6$.

In the next section we introduce notation, prove two small lemmas and state the main lemma. In Section 3 we prove Theorem 1.1 modulo the main lemma. In Section 4 we derive linear-time algorithms for acyclic coloring of graphs with bounded maximum degree. In the last section we give the proof of the main lemma.

\section{Preliminaries}

Let $G$ be a graph. A partial coloring of $G$ is a coloring of some subset of the vertices of $G$. A partial acyclic coloring is then a proper partial coloring of $G$ containing no bicolored cycles. 
Given a partial coloring $f$ of $G$, a vertex $v$ is

(a) rainbow if all colored neighbors of $v$ have distinct colors;

(b) almost rainbow if there is a color $c$ such that exactly two neighbors of $v$ are colored with $c$ and all other colored neighbors of $v$ have distinct colors;

(c) admissible if it is either rainbow or almost rainbow;

(d) defective if $v$ is an uncolored almost rainbow vertex such that at least one of the two of its neighbors receiving the same color is admissible.

A partial acyclic coloring $f$ of a graph $G$ is rainbow if $f$ is a partial acyclic coloring of $G$ such that every uncolored vertex is rainbow.

A partial acyclic coloring $f$ of a graph $G$ is admissible if either $f$ is rainbow or one vertex is defective and all other uncolored vertices are rainbow. In these terms, a coloring is rainbow if it is admissible and has no defective vertices. Note that both, rainbow and admissible colorings are partial acyclic colorings where additional restrictions are put only on uncolored vertices. The advantage of using admissible colorings is that they provide a stronger induction condition that places additional restrictions only on coloring of neighbors of uncolored vertices. So, the fewer uncolored vertices remain, the weaker these additional restrictions are.

All colorings in this section will be from the set $\{1,2, \ldots, 7\}$.

Lemma 2.1. Let $v$ be a vertex of degree 4 in a graph $G$ with $\Delta(G) \leq 5$. Let $f$ be an admissible (respectively, rainbow) coloring in which $v$ is colored with color $c_{1}$, each of the neighbors of $v$ is colored, and exactly 3 colors appear on the neighbors of $v$. If at least one of the two neighbors of $v$ receiving the same color and one of the other two neighbors of $v$ each have a second (i.e., distinct from $v$ ) neighbor with color $c_{1}$, then we can recolor $v$ and at most one of its neighbors so that the coloring remains admissible (respectively, rainbow). In particular, the new partial acyclic coloring has no new defective vertices. Moreover, if we need to recolor a vertex other than $v$, then we may choose a vertex with 5 colored neighbors and recolor it with a color incident to $v$ in $f$.

Proof. Let $N(v)=\left\{z_{1}, z_{2}, z_{3}, z_{4}\right\}, f\left(z_{1}\right)=f\left(z_{2}\right)=c_{2}, f\left(z_{3}\right)=c_{3}, f\left(z_{4}\right)=c_{4}$. Let $z_{2}$ and $z_{3}$ be the neighbors of $v$ with colors $c_{2}$, and $c_{3}$ that are also adjacent to another vertex of color $c_{1}$. We may assume that $z_{2}$ is adjacent to a vertex of color $c_{5}$, since otherwise when we recolor $v$ with $c_{5}$, no bicolored cycles appear and the coloring remains admissible (respectively, rainbow). Similarly, we may assume that $z_{2}$ is adjacent to vertices of colors $c_{6}$ and $c_{7}$. Then we may recolor $z_{2}$ with $c_{3}$ and repeat the above argument to get that $z_{3}$ also is adjacent to vertices with colors $c_{5}, c_{6}$, and $c_{7}$. In this case, we may change the original coloring by recoloring $z_{3}$ with $c_{2}$ and $v$ with $c_{3}$. So, in this case only $v$ and $z_{3}$ change colors. Note that either only $v$ changes its color, or $z_{2}$ receives color $c_{3}$, or $z_{3}$ receives color $c_{2}$.

For partial colorings $f$ and $f^{\prime}$ of a graph $G$, we say that $f^{\prime}$ is larger than $f$ if it colors more vertices.

Lemma 2.2. Let $v$ be a vertex of degree 4 in a graph $G$ with $\Delta(G) \leq 5$. Let $f$ be a rainbow coloring in which $v$ is colored with color $c_{1}$, the neighbors $z_{1}, z_{2}$, and $z_{3}$ of $v$ receive the distinct colors $c_{2}, c_{3}$, and $c_{4}$, the neighbor $z_{4}$ of $v$ is an uncolored rainbow vertex. Then either $G$ has a rainbow coloring $f_{1}$ that colors the same vertices and differs from $f$ only at $v$, or $G$ has a rainbow coloring $f^{\prime}$ larger than $f$. Moreover, if the former does not hold, 
then $z_{4}$ has degree 5 and exactly one uncolored neighbor, say $z_{4,4}$, and we can choose the larger coloring $f^{\prime}$ so that all the following are true:

\section{Every vertex colored in $f$ is still colored.}

2. Vertex $z_{4}$ is colored.

3. The only uncolored vertex apart from $z_{4}$ that may get colored is $z_{4,4}$, and it does only if it has neighbors of colors $c_{1}, c_{2}, c_{3}$, and $c_{4}$.

4. Apart from $v$, only one vertex $w$ may change its color, and if it does, then (a) $w$ is a neighbor of $z_{4}$, (b) $w$ has four colored neighbors, (c) it changes a color in $\left\{c_{5}, c_{6}, c_{7}\right\}$ to another color in $\left\{c_{5}, c_{6}, c_{7}\right\}$, and $(d) z_{4}$ gets the former color of $w$. In particular, $v$ is admissible in $f^{\prime}$.

Proof. Let $v, z_{1}, z_{2}, z_{3}$, and $v_{4}$ be as in the hypothesis. We may assume that $z_{4}$ is adjacent to a vertex $z_{4,1}$ of color $c_{5}$ : otherwise, since $v_{4}$ is rainbow, when we recolor $v$ with $c_{5}$, the new coloring will be rainbow. Similarly, we may assume that $z_{4}$ is adjacent to vertices $z_{4,2}$, and $z_{4,3}$ of colors $c_{6}$ and $c_{7}$. If $z_{4}$ has no other neighbors, then we can recolor $v$ with $c_{5}$ and color $z_{4}$ with $c_{1}$. So, assume that $z_{4}$ has the fifth neighbor, $z_{4,4}$. If $z_{4,4}$ is colored, then $f\left(z_{4,4}\right) \in\left\{c_{2}, c_{3}, c_{4}\right\}$, since $z_{4}$ is rainbow. In this case, we let $f^{\prime}\left(z_{4}\right)=c_{1}$ and $f^{\prime}(v)=c_{5}$. So, we may assume that $z_{4,4}$ is not colored. If $z_{4,4}$ has no neighbor of color $c_{2}$, then coloring $z_{4}$ with $c_{2}$ leaves the coloring rainbow and makes it larger than $f$. Thus, we may assume that $z_{4,4}$ has a neighbor of color $c_{2}$ and similarly neighbors of colors $c_{3}$ and $c_{4}$. If $z_{4,4}$ has no neighbor of color $c_{1}$, then we let $f^{\prime}\left(z_{4}\right)=c_{1}$ and $f^{\prime}(v)=c_{5}$. So, let $z_{4,4}$ have such a neighbor.

If $z_{4,1}$ has no neighbor of color $c_{2}$, then by coloring $z_{4}$ with $c_{2}$ and $z_{4,4}$ with $c_{5}$, we get a rainbow coloring larger than $f$. So, we may assume (by symmetry) that $z_{4,1}$ has neighbors of colors $c_{2}, c_{3}, c_{4}$. If $z_{4,1}$ has no neighbor of color $c_{1}$, then we let $f^{\prime}\left(z_{4}\right)=c_{1}$, $f^{\prime}\left(z_{4,4}\right)=c_{5}$, and $f^{\prime}(v)=c_{6}$. Finally, if $z_{4,1}$ also has a neighbor of color $c_{1}$, then we let $f^{\prime}\left(z_{4,1}\right)=c_{6}$ and $f^{\prime}\left(z_{4}\right)=c_{5}$.

The next lemma is our main lemma. We will use it in the next section and prove in Section 5.

Lemma 2.3. Let $f$ be an admissible partial coloring of a 5-regular graph $G$. Then $G$ has a rainbow coloring $f^{\prime}$ that colors at least as many vertices as $f$.

\section{Proof the the Theorem}

For convenience, we restate Theorem 1.1.

Theorem. Every graph with maximum degree 5 has an acyclic 7 -coloring.

Proof. Let $G$ be such a graph. If $G$ is not 5-regular, form $G^{\prime}$ from two disjoint copies of $G$ by adding for each $v \in V(G)$ of degree less than 5 an edge between the copies of $v$. Repeating this process at most five times gives a 5 -regular graph $G^{*}$ containing $G$ as a subgraph. Since an acyclic 7 -coloring of $G^{*}$ yields an acyclic 7-coloring of its subgraph $G$, we may assume that $G$ is 5 -regular.

Let $f$ be an admissible coloring of $G$ from the set $\{1,2, \ldots, 7\}$ with the most colored vertices. By Lemma 2.3, we may assume that $f$ is rainbow. 
Let $H$ be the subgraph of $G$ induced by the vertices left uncolored by $f$. Let $x$ be a vertex of minimum degree in $H$. We consider several cases according to the degree $d_{H}(x)$.

Case 1: $d_{H}(x)=0$. Since $f$ is rainbow, any color in $\{1,2, \ldots, 7\}-f\left(N_{G}(x)\right)$ can be used to color $x$ contradicting the maximality of $f$.

Case 2: $d_{H}(x)=1$. Since $f$ is rainbow, we may assume that $x$ is adjacent to vertices of colors $1,2,3$, and 4 . Let $y$ be the uncolored neighbor of $x$. Since $y$ is rainbow, coloring $x$ with 5 gives either a rainbow coloring or an admissible coloring with the defective vertex $y$ having the admissible neighbor $x$, a contradiction to the maximality of $f$.

Case 3: $d_{H}(x)=2$. We may assume that $x$ is adjacent to vertices with colors $1,2,3$, and two uncolored vertices $y_{1}$ and $y_{2}$. Since in our case $y_{1}$ is adjacent to at most 3 colored vertices, some color $c \in\{4,5,6,7\}$ does not appear on the neighbors of $y_{1}$. Coloring $x$ with $c$ then yields either a rainbow coloring, or an admissible coloring with defective vertex $y_{2}$ and its admissible neighbor $x$, a contradiction to the maximality of $f$.

Case 4: $d_{H}(x)=3$. We may assume that $x$ is adjacent to vertices of colors 1 and 2 . By the choice of $x$, each uncolored vertex of $G$ has at most 2 colored neighbors. Since the three uncolored neighbors of $x$ have at most 6 colored neighbors in total, some color $c \in\{3,4,5,6,7\}$ is present at most once among these 6 neighbors. Then coloring $x$ with $c$ again yields an admissible coloring, a contradiction to the maximality of $f$.

Case 5: $d_{H}(x) \geq 4$. Since each vertex of $G$ has at most one colored neighbor, at most 5 colors are used in the second neighborhood of $x$. Hence $x$ may be colored to give a rainbow coloring with more colored vertices.

We conclude that $H$ is empty and that $f$ is an acyclic 5-coloring of $G$.

\section{Algorithms}

Theorem 4.1. There exists a linear time algorithm for finding an acyclic 7-coloring of a graph with maximum degree 5.

Proof. The proof of the Theorem 1.1, along with Lemmas 2.1-2.3 gives an algorithm. In order to control the efficiency of the algorithm we make the following modification: whenever the proof checks whether a vertex $v$ is in a two-colored cycle, we check only for such a cycle of length at most 12 , and if we do not find such a short cycle, then check whether two bicolored paths of length 6 leave $v$. This is enough, since the existence of such paths already makes the proofs of Theorem 1.1 and all the lemmas work. So, we need only to consider a bounded (at most $5^{6}$ ) number of vertices around our vertex. It then suffices to compute the running time of this algorithm. Let $n$ be the number of vertices in $G$. The process of creating a 5-regular graph takes $O(n)$ time since we apply this process at most 5 times, each time on at most $2^{5} n$ vertices, each of degree at most 5 . We may now assume that $G$ is a 5 -regular graph. We then create and maintain 6 databases $D_{j}, j=0,1, \ldots, 5$ (say doubly linked lists), each for the set of vertices with degree $j$ in the current $H$. At the beginning, all vertices are in $D_{5}$, and it is possible to update the databases in a constant amount of time each time a vertex gains or loses a colored neighbor. Since there are at most $2^{5} n$ possible searches for a vertex with the minimum number of uncolored neighbors, all the searches and updates will take $O(n)$ time. Note that the processes of Lemma 2.1 and Lemma 2.2 also take a constant amount of time to complete. Observe that each of the cases in Lemma 2.3 either finds a rainbow coloring, or finds an admissible coloring with more colored vertices, or reduces to a previous case in an amount of time bounded by a constant. Also when Lemma 2.3 processes a defective vertex, it yields either a rainbow coloring, or 
a larger admissible coloring and the next defective vertex in a constant time. Finally, since we start from an uncolored graph and color each additional vertex in a constant time, the implied algorithm colors all vertices in $O(n)$ time.

For a partial coloring $f$ of a graph $G$ and a vertex $v \in V(G)$, we say that $u \in V(G)$ is $f$-visible from $v$, if either $v u \in E(G)$ or $v$ and $u$ have a common uncolored neighbor.

Theorem 4.2. For every fixed $r$, there exists a linear (in $n$ ) algorithm finding an acyclic coloring for any n-vertex graph $G$ with maximum degree $r$ using at most $1+\left\lfloor\frac{(1+r)^{2}}{4}\right\rfloor$ colors.

Proof. We start from the partial coloring $f_{0}$ that has no colored vertices, and for $i=$ $1, \ldots, n$ at Step $i$ obtain a rainbow partial acyclic coloring $f_{i}$ from $f_{i-1}$ by coloring one more vertex (without recoloring). The algorithm proceeds as follows: at Step $i$, choose an uncolored vertex $v_{i}$ with the most colored neighbors. Greedily color $v_{i}$ with a color $\alpha_{i}$ in $C:=\left\{1, \ldots, 1+\left\lfloor\frac{(1+r)^{2}}{4}\right\rfloor\right\}$ that is distinct from the colors of all vertices $f_{i-1}$-visible from $v_{i}$. We claim that we always can find such $\alpha_{i}$ in $C$.

Suppose that at Step $i, v_{i}$ has exactly $k$ colored neighbors. Then it has at most $r-k$ uncolored neighbors, and each of these uncolored neighbors has at most $k$ colored neighbors. So, the total number of vertices $f_{i-1}$-visible from $v_{i}$ is at most

$$
k+(r-k) k=k(r+1-k) \leq\left\lfloor\frac{(r+1)^{2}}{4}\right\rfloor=|C|-1,
$$

and we can find a suitable color $\alpha_{i}$ for $v_{i}$.

It now suffices to show that for each $i$, the coloring $f_{i}$ is rainbow and acyclic. For $f_{0}$, this is obvious. Assume now that $f_{i-1}$ is rainbow and acyclic. Since $v_{i}$ is rainbow in $f_{i-1}$, coloring it with $\alpha_{i}$ does not create bicolored cycles. Thus, $f_{i}$ is acyclic. Also since $\alpha_{i}$ is distinct from the colors of all vertices $f_{i-1}$-visible from $v_{i}, f_{i}$ is rainbow.

For the runtime, note that at Step $i$ the algorithm considers only $v_{i}$ and vertices at distance at most 2 from $v_{i}$. As in the proof of Theorem 4.1, it is sufficient to maintain $r+1$ databases each containing all vertices with a given number of colored neighbors. This allows a constant time search for a vertex with the greatest number of colored neighbors. Moving a vertex as its number of colored neighbors changes takes a constant amount of time. Choosing and coloring $v_{i}$ together with updating the databases then takes $O\left(r^{2}\right)$ time. Hence the running time of the algorithm is at most $c_{r} n$, where $c_{r}$ depends on $r$.

\section{Proof of Lemma 2.3}

We will prove that under the conditions of the lemma, either its conclusion holds or there is an admissible coloring $f^{\prime \prime}$ larger than $f$. Since $G$ is finite, repeating the argument eventually yields either an acyclic coloring of the whole $G$ or a rainbow coloring. In both cases we do not have defective vertices.

Let $H$ be the subgraph of $G$ induced by the uncolored vertices. Let $x$ be the sole defective vertex under $f$ and let $y_{1}, y_{2}, \ldots, y_{5}$ be its neighbors. By the definition of a defective vertex, $x$ has two neighbors of the same color. We will assume that $f\left(y_{1}\right)=$ $f\left(y_{2}\right)=1$ and that $y_{1}$ is admissible. When more then two neighbors of $x$ are colored, we assume for $i=3,4,5$ that if $y_{i}$ is colored, then $f\left(y_{i}\right)=i-1$. Also for $i=1, \ldots, 5$, the four neighbors of $y_{i}$ distinct from $x$ will be denoted by $y_{i, 1}, \ldots, y_{i, 4}$ (some vertices will 
have more than one name, since they may be adjacent to more than one $y_{i}$ ). We consider several cases depending on $d_{H}(x)$.

Case 1: $d_{H}(x)=0$. First we try to color $x$ with colors 5,6 , and 7 . If this is not allowed, then for $j=5,6,7, G$ has a $1, j$-colored $y_{1}, y_{2}$-path. This forces that both of $y_{1}$ and $y_{2}$ have neighbors with colors 5,6 , and 7 , each of which is adjacent to another vertex of color 1 . In particular, both $y_{1}$ and $y_{2}$ are admissible. For $i=1,2$ and $j=1,2,3$, we suppose that $f\left(y_{i, j}\right)=j+4$ and $y_{i, j}$ is adjacent to another vertex of color 1 .

Case 1.1: For some $i \in\{1,2\}, y_{i, 4}$ is colored and $f\left(y_{i, 4}\right) \notin\{5,6,7\}$. By symmetry, we may assume that $i=1$ and $f\left(y_{1,4}\right)=2$. Recolor $y_{1}$ with 3 and call the new admissible coloring $f^{\prime}$. If we can now recolor $y_{2}$ so that the resulting coloring $f^{\prime \prime}$ is rainbow on $G-x y_{2}-x y_{1}$ or the only defective vertex in $f^{\prime \prime}$ on $G-x y_{2}-x y_{1}$ is $y_{2,4}$, then we do this recoloring and color $x$ with 1 . Since $y_{1}$ and $y_{2}$ have no neighbors of color 1 apart from $x$, we obtained an admissible coloring of $G$ larger than $f$. If we cannot recolor $y_{2}$ to get such a coloring, then $y_{2,4}$ is colored with a color $c \in\{5,6,7\}$. Moreover, in this case by Lemma 2.1 applied to $y_{2}$ in coloring $f^{\prime}$ of $G-x y_{2}-x y_{1}$, we can change the colors of only $y_{2}$ and some $y \in\left\{y_{2,1}, y_{2,2}, y_{2,3}, y_{2,4}\right\}$ to get an admissible coloring $f_{1}$ of $G-x y_{2}-x y_{1}$. Moreover, by Lemma $2.1, f_{1}(y) \in\{5,6,7\}$. Then by coloring $x$ with 1 we obtain a rainbow coloring of $G$, as above.

Case 1.2: $y_{1,4}$ is not colored. By Lemma 2.2 for vertex $y_{1}$ in $G-x y_{1}$, either $G-x y_{1}$ has a rainbow coloring $f^{\prime}$ that differs from $f$ only at $y_{1}$ (in which case by symmetry, we may assume that $f^{\prime}\left(y_{1}\right)=3$ and proceed further exactly as in Case 1.1), or $G-x y_{1}$ has a larger rainbow coloring $f^{\prime}$ satisfying statements 1)-4) of Lemma 2.2. In particular, by 4), none of $y_{2}, y_{3}, y_{4}, y_{5}$ changes its color and $y_{1}$ remains admissible. This finishes Case 1.2.

By the symmetry between $y_{1}$ and $y_{2}$, the remaining subcase is the following.

Case 1.3: $f\left(y_{1,4}\right)=5$ and $f\left(y_{2,4}\right)=c \in\{5,6,7\}$. By Lemma 2.1 applied to $y_{1}$ in $G-x y_{1}$, we can recolor $y_{1}$ and at most one other vertex (a neighbor of $y_{1}$ ) to obtain another admissible coloring $f^{\prime}$. If $f^{\prime}\left(y_{1}\right) \in\{5,6,7\}$, then $f^{\prime}$ is a rainbow coloring, as claimed. So, we may assume that $f^{\prime}\left(y_{1}\right)=c_{1} \in\{2,3,4\}$. If all the colors $5,6,7$ are present on neighbors of $y_{2}$, then again by Lemma 2.1 (applied now to $y_{2}$ in coloring $f^{\prime}$ of $\left.G-x y_{2}\right), G$ has an admissible coloring $f^{\prime \prime}$ that differs from $f^{\prime}$ only at $y_{2}$ and maybe at one neighbor of $y_{2}$. Then coloring $x$ with 1 we get a rainbow coloring. So, some color in $\{5,6,7\}$ is not present in $f^{\prime}\left(N\left(y_{2}\right)\right)$. By Lemma 2.1, this may happen only if $y_{1,1}$ is a common neighbor of $y_{1}$ and $y_{2}$, and $c=f\left(y_{2,4}\right) \neq 5$. In particular, in this case, $y_{1,1}$ has neighbors of colors 1 (they are $y_{1}$ and $y_{2}$ ), 2,3, and 4 . Since $c \neq 5$, we may assume that $c=6$. By the symmetry between $y_{1}$ and $y_{2}$, we conclude that, in $f$, vertex $y_{2,2}$ also is a common neighbor of $y_{1}$ and $y_{2}$ and has neighbors of colors 1 (they are $y_{1}$ and $y_{2}$ ), 2, 3, and 4. Returning to coloring $f^{\prime}$, we see that $y_{2}$ has no neighbors of color 5 , and its neighbors $y_{1,1}$ (formerly of color 5 ) and $y_{2,2}$ (by the previous sentence) also have no neighbors of color 5 . So, recoloring $y_{2}$ with 5 yields an admissible coloring of $G$. Now coloring $x$ with 1 creates a larger rainbow coloring.

Case 2: $d_{H}(x)=1$. We first try to color $x$ with 4 . If no bicolored cycle is formed, then either we have a rainbow coloring or an admissible coloring with defective vertex $y_{5}$ and an admissible neighbor $x$. Hence we may assume that coloring $x$ with 4 creates a bicolored cycle. This then gives each of $y_{1}$ and $y_{2}$ a neighbor of color 4 . A similar argument gives each of $y_{1}$ and $y_{2}$ a neighbor of color 5,6 , and 7 , i.e., both $y_{1}$ and $y_{2}$ are rainbow. Recoloring $y_{1}$ with color 2 allows us to repeat the argument at $y_{3}$. Then $y_{3}$ also has neighbors of each of the colors $4,5,6$, and 7 . If $y_{5}$ has no neighbor of color 
2 , then recoloring (in the original coloring $f$ ) $y_{3}$ with 1 , and coloring $x$ with 2 yields a rainbow coloring. So, by the symmetry between colors 1,2 , and 3 , we may assume that for $i \in\{1,2,3\}, f\left(y_{5, i}\right)=i$. Since $y_{5}$ is rainbow, by the symmetry between colors $4,5,6$, and 7 , we may assume that either $f\left(y_{5,4}\right)=4$, or $y_{5,4}$ is not colored. In both cases, recolor (in the original coloring $f$ ) $y_{3}$ with 1 , color $x$ with 2 and $y_{5}$ with 5 . We get an admissible coloring larger than $f$, where only $y_{5,4}$ may be defective.

Case 3: $d_{H}(x)=3$. If one of the uncolored neighbors $y_{3}, y_{4}, y_{5}$ (say, $y_{3}$ ) of $x$ has 4 colored neighbors, then we may color $y_{3}$ with some $c \notin f\left(N\left(y_{3}\right)\right) \cup\{1\}$ and thus create an admissible coloring larger than $f$. Hence we may assume that each of $y_{3}, y_{4}$, and $y_{5}$ has at most 3 colored neighbors.

Case 3.1: One of $y_{1}$ and $y_{2}$ has three neighbors of different colors such that each of these neighbors has another neighbor of color 1 . Suppose for example that for $j=1,2,3$, $f\left(y_{1, j}\right)=1+j$ and $y_{1, j}$ has another neighbor of color 1 . If $y_{1}$ has a fourth color, say $c$, in its neighborhood, then we recolor $y_{1}$ with a color $c^{\prime} \notin\{1, c, 5,6,7\}$ and get a rainbow coloring of $G$. Suppose now that color $c \in\{5,6,7\}$ appears twice on $N\left(y_{1}\right)$. Then by Lemma 2.1 applied to $y_{1}$ in $G-x y_{1}$, we can change the color of $y_{1}$ and at most one other vertex that is a neighbor of $y_{1}$ not adjacent to uncolored vertices to get another rainbow coloring of $G-x y_{1}$. Then this coloring will also be a rainbow coloring of $G$. Finally, suppose that $y_{1}$ has an uncolored neighbor $y_{1,4}$. Applying Lemma 2.2 to $y_{1}$ in $G-x y_{1}$ we either recolor only $y_{1}$ and get a rainbow coloring of $G$ (finishing the case), or obtain a rainbow coloring $f^{\prime}$ of $G-x y_{1}$ larger than $f$ satisfying the conclusions of the lemma. Since each of $y_{3}, y_{4}$ and $y_{5}$ has at least two neighbors left uncolored by $f$, none of them may play role of $z_{4}$ or $z_{4,4}$ in Lemma 2.2 when they get colored. Then $f^{\prime}$ is an admissible coloring of $G$ where only $x$ could be a defective vertex with admissible neighbor $v$. This proves Case 3.1.

Let $T$ be the set of colors $c$ such that more than one of the vertices $y_{3}, y_{4}$ and $y_{5}$ has a neighbor of color $c$. Since $y_{3}, y_{4}$ and $y_{5}$ have in total at most 9 colored neighbors, $|T| \leq 4$.

Case 3.2: $|T| \leq 3$. By symmetry, we may assume that $T \subseteq\{2,3,4\}$. If coloring $x$ with $c \in\{5,6,7\}$ does not create a bicolored cycle, then it will yield an admissible coloring larger than $f$. So, we may assume that each of $y_{1}$ and $y_{2}$ has in its neighborhood vertices of colors 5,6 , and 7 , each of which is adjacent to another vertex of color 1 . So, we have Case 3.1.

Case 3.3: $|T|=4$. Let $T=\{2,3,4,5\}$. As in Case 3.1, we may assume that each of $y_{1}$ and $y_{2}$ is adjacent to vertices of colors 6 and 7 , each of which have another neighbor of color 1 .

Let $y_{3}$ have exactly 3 colored neighbors labeled $y_{3,1}, y_{3,2}, y_{3,3}$ with colors $2,3,4$. Let $y_{3,4}$ be the uncolored neighbor of $y_{3}$. Then if $y_{3,4}$ has no neighbor of color 5 , we may color $y_{3}$ with 5 to get a new admissible coloring. Hence $y_{3,4}$ is adjacent to a vertex of color 5 . Similarly, $y_{3,4}$ has neighbors of color 6 and 7 . By symmetry, we may assume that a vertex of color 2 is adjacent to at most one of $y_{4}$ and $y_{5}$.

Case 3.3.1: $y_{3,4}$ has no neighbor of color 1 . We try to color $y_{3}$ with 1 and $x$ with 2 . If this does not produce a new admissible coloring, then one of $y_{1}$ or $y_{2}$, say $y_{1}$, has a neighbor of color 2 that is adjacent to another vertex of color 1. So, we again get Case 3.1.

Case 3.3.2: $y_{3,4}$ has a neighbor of color 1 . If $y_{3,1}$ has no neighbor of color 1 , then we again try to color $y_{3}$ with 1 and $x$ with 2 , but also color $y_{3,4}$ with 2 . Then we simply repeat the argument of Case 3.3.1. So, suppose that $y_{3,1}$ has a neighbor of color 1 . If $y_{3,1}$ has no neighbor of some color $\alpha \in\{5,6,7\}$, then we color $y_{3,4}$ with 2 and $y_{3}$ with $\alpha$. Thus $y_{3,1}$ 


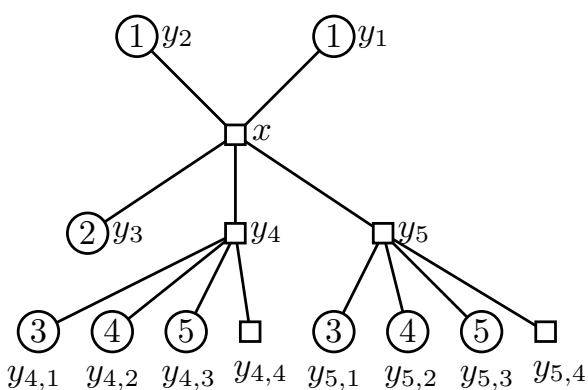

Case 4.3.

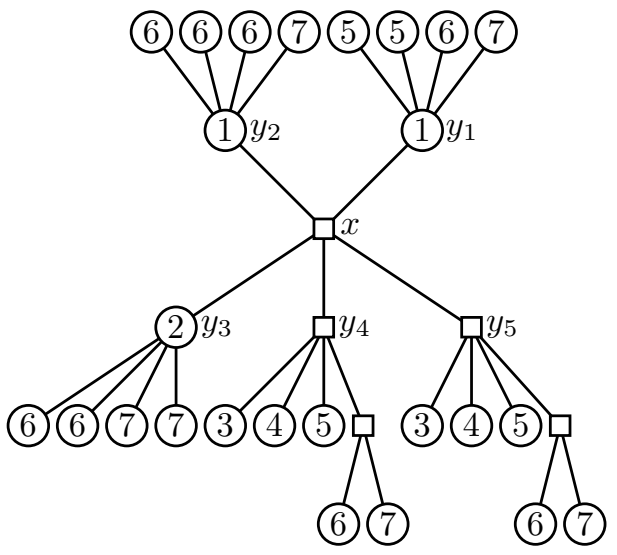

Case 4.3.4.3.

Figure 1: Cases 4.3 and 4.3.4.3 from the proof of Lemma 2.3.

has neighbors of colors $1,5,6,7$. Then we recolor $y_{3,1}$ with 3 and color $y_{3}$ with 2 .

Case 4: $d_{H}(x)=2$. As at the beginning of Case 3, we conclude that each of the uncolored vertices $y_{4}$ and $y_{5}$ has at least one uncolored neighbor besides $x$.

Let $B$ be the set of colors appearing in the neighborhoods of both, $y_{4}$ and $y_{5}$. By the previous paragraph, $|B| \leq 3$.

Case 4.1: $|B| \leq 1$. We may assume that $\{4,5,6,7\} \cap B=\emptyset$. Try to color $x$ with 4. By the definition of $B$, either a two-colored cycle appears, or we get a new admissible coloring larger than $f$. Hence we may assume that coloring $x$ with 4 creates a bicolored cycle. Since this cycle necessarily goes through $y_{1}, y_{1}$ is adjacent to a vertex with color 4. Similarly, $y_{1}$ is adjacent to vertices with colors 5,6 , and 7 . Then recoloring $y_{1}$ with 3 yields a rainbow coloring of $G$.

Case 4.2: $|B|=2$. If $1 \in B$ or $2 \in B$, then the argument of Case 4.1 holds. Assume that $B=\{3,4\}$. Similarly to Case 4.1 , we may assume that for $i=1,2$ and $j=1,2,3$, $y_{i}$ is adjacent to a vertex $y_{i, j}$ of color $j+4$ that is adjacent to another vertex of color 1 (in particular, $y_{1}$ and $y_{2}$ may have a common neighbor of color $j+4$ ).

If $y_{1}$ is rainbow, then uncoloring $y_{1}$ and coloring $x$ with 7 gives Case 1 or Case 2. Thus we may assume that $y_{1}$ and (by symmetry) $y_{2}$ are not rainbow. So, we may assume that for $i=1,2$, the fourth neighbor $y_{i, 4}$ of $y_{i}$ distinct from $x$ has color $c_{i} \in\{5,6,7\}$. By symmetry, we may assume that $c_{1}=5$. Similarly to Case 1.3 , by Lemma 2.1 applied to $y_{1}$ in $G-x y_{1}$, we can recolor $y_{1}$ and at most one other vertex (a neighbor of $y_{1}$ ) to obtain another rainbow coloring $f^{\prime}$ of $G-x y_{1}$. If $f^{\prime}\left(y_{1}\right) \in\{3,4,5,6,7\}$, then $f^{\prime}$ is a rainbow coloring of $G$, as claimed. So, we may assume that $f^{\prime}\left(y_{1}\right)=2$. Now practically repeating the argument of Case 1.3, we find a promised coloring.

Case 4.3: $|B|=3$ (see Figure 1). If $2 \in B$, then we can repeat the argument of Case 4.2 for $B^{\prime}=B-\{2\}$. Hence we may assume that $B \subseteq\{1,3,4,5,6,7\}$.

Case 4.3.0: $1 \in B$. Let $B=\{1,3,4\}$. Then some color in $\{5,6,7\}$, say 7 , is not present on $N\left(y_{4}\right) \cup N\left(y_{5}\right)$. Again, we may assume that for $i=1,2$ and $j=1,2,3, y_{i}$ is 
adjacent to a vertex $y_{i, j}$ of color $j+4$ that is adjacent to another vertex of color 1 . If $y_{1}$ is rainbow, then we may uncolor $y_{1}$ and color $x$ with 7 to get Case 1 or Case 2. Suppose now that $y_{1}$ and $y_{2}$ are not rainbow. By Lemma 2.1 applied to $y_{1}$ in $G-x y_{1}$, we can recolor $y_{1}$ and at most one other vertex (a neighbor of $y_{1}$ ) to obtain another admissible coloring $f^{\prime}$. If $f^{\prime}\left(y_{1}\right) \in\{3,4,5,6,7\}$, then $f^{\prime}$ is a rainbow coloring, as claimed. So, we may assume that $f^{\prime}\left(y_{1}\right)=2$. But then we can use the argument of Case 4.2 with the roles of $y_{3}$ and $y_{2}$ switched. This proves Case 4.3.0.

So, from now on, $B=\{3,4,5\}$. For $i=4,5$ and $j=1,2,3$, let $y_{i, j}$ be the neighbor of $y_{i}$ of color $j+2$. We write the neighbor, since $y_{4}$ and $y_{5}$ are rainbow. As observed at the beginning of Case $4, y_{4}$ and $y_{5}$ each have another uncolored neighbor, call them $y_{4,4}$ and $y_{5,4}$. In particular, $y_{4}$ and $y_{5}$ have no neighbors colored with 6 or 7 . If $x$ can be colored with either of 6 or 7 without creating a two-colored cycle, then we obtain a rainbow coloring. Hence we assume that for $i=1,2$ and $j=1,2, f\left(y_{i, j}\right)=j+5$ and $y_{i, j}$ has a neighbor of color 1 distinct from $y_{i}$.

Case 4.3.1: One of $y_{1}$ or $y_{2}$, say $y_{1}$, is rainbow. If $y_{4,4}$ has no neighbor of color $c \in\{6,7\}$, then we can color $y_{4}$ with $c$, a contradiction to the maximality of $f$. If $y_{4,4}$ has no neighbor of color $c^{\prime} \in\{1,2\}$, then by uncoloring $y_{1}$ and coloring $y_{4}$ with $c^{\prime}$ and $x$ with 6 , we obtain an admissible coloring larger than $f$. So, $f\left(N\left(y_{4,4}\right)\right)=\{1,2,6,7\}$. Then we may color $y_{4,4}$ with 3 and uncolor $y_{1}$ to get a new admissible coloring as large as $f$ with one defective vertex $y_{4}$, for which Case 2 holds. This finishes Case 4.3.1.

So, below $y_{1}$ and $y_{2}$ are not rainbow and hence each of them is adjacent to at least three colored vertices.

Case 4.3.2: One of $y_{1}$ or $y_{2}$, say $y_{1}$, is adjacent to an uncolored vertex $y_{1,4} \neq x$. We may assume that $f\left(y_{1,1}\right)=f\left(y_{1,2}\right)=6$ and $f\left(y_{1,3}\right)=7$. First, we try to color $x$ with 7 and $y_{1}$ with 3 . Since the new coloring has at most one defective vertex, we may assume that a two-colored cycle is created. Hence each of $y_{1,1}$ and $y_{1,2}$ is adjacent to a vertex of color 3 . The same argument gives these vertices neighbors of colors 4 and 5 . Recall that one of $y_{1,1}$ and $y_{1,2}$, say $y_{1,1}$, has another neighbor of color 1 . Then recoloring $y_{1,1}$ with 2 gives an admissible coloring in which $y_{1}$ is rainbow. Hence Case 4.3.1 applies to this new coloring.

So, from now on each of $y_{1}$ and $y_{2}$ has 4 colored neighbors. Since $y_{1}$ is admissible we may assume w.l.o.g. that $y_{1}$ is adjacent either to the colors $5,6,6,7$ or the colors $5,5,6,7$.

Case 4.3.3: $y_{1}$ has one neighbor of color 5 and three neighbors with colors 6 or 7 . We may assume that $f\left(y_{1,1}\right)=5, f\left(y_{1,2}\right)=f\left(y_{1,3}\right)=6$, and $f\left(y_{1,4}\right)=7$. If coloring $y_{1}$ with 3 or 4 yields an admissible coloring, then we are done; so we may assume that a twocolored cycle is formed in each case. It follows that each of $y_{1,2}$ and $y_{1,3}$ has neighbors colored with 3 and 4 . By the symmetry between $y_{1,2}$ and $y_{1,3}$, we may assume that $y_{1,3}$ has a neighbor of color 1 other than $y_{1}$. If $y_{1,3}$ is almost rainbow, then we can uncolor it, recolor $y_{1}$ with 3 , and color $x$ with 7: this will give an admissible coloring with the same number of colored vertices as in $f$, and the only defective vertex $y_{1,3}$. Then either Case 1 or Case 2 applies to this new coloring. Hence we may assume that $y_{1,3}$ has two neighbors other than $y_{1}$ that receive the same color. Then since $y_{1,3}$ has no neighbor of color $2, y_{1}$ may now be recolored with color 2 without creating a bicolored cycle. Repeating the above argument we derive that $y_{1,2}$ has neighbors of colors 2,3 , and 4 , and one of these colors appears twice on $N\left(y_{1,2}\right)-y_{1}$. By Lemma 2.1 applied to $y_{1,3}$ in the graph $G-y_{1,3} y_{1}$ for the original coloring, we can change its color and the color of at most one other vertex (that is a neighbor of $y_{1,3}$, all of whose neighbors are colored) to get an admissible coloring of 
$G-y_{1,3} y_{1}$. Since $y_{2}$ and $y_{3}$ are adjacent to the uncolored vertex $x$, their colors are not changed. If $y_{1,3}$ receives color 1 , then we recolor $y_{1}$ with 3 and get a rainbow coloring of $G$. If $y_{1,3}$ receives a color other than 1 , then we color $x$ with 6 and again get a rainbow coloring of $G$.

Case 4.3.4: $y_{1}$ has two neighbors of color 5 (see Figure 1). We may assume that $f\left(y_{1,1}\right)=f\left(y_{1,2}\right)=5, f\left(y_{1,3}\right)=6$, and $f\left(y_{1,4}\right)=7$. If $y_{1}$ can be recolored with either 3 or 4 , this would give a rainbow coloring $f^{\prime}$. Hence we assume that both of $y_{1,1}$ and $y_{1,2}$ are adjacent to vertices with colors 3 and 4 .

Case 4.3.4.1: One of $y_{1,1}$ or $y_{1,2}$, say $y_{1,1}$, is rainbow. Then uncoloring $y_{1,1}$ and coloring $y_{1}$ with 3 and $x$ with 7 yields either a rainbow coloring $f^{\prime}$ or a new admissible coloring (with the same number of colored vertices) with the defective vertex $y_{1,1}$ and admissible colored neighbor $y_{1}$. In the former case, we are done. In the latter, if one of the previous cases occurs, then we are done again. So, we may assume that Case 4.3.4 occurs. By the symmetry between colors 3 and 4 , we may assume that apart from $y_{1}$, vertex $y_{1,1}$ has a neighbor of color 3 , a neighbor of color 4 , and two uncolored neighbors, say $z_{1}$ and $z_{2}$, each of whose has another uncolored neighbor and 3 colored neighbors. Moreover, the same 3 colors appear on the neighborhoods of $z_{1}$ and $z_{2}$, and since Case 4.3 .4 holds, by the symmetry between colors 6 and 7 , both of them are among these 3 colors. Then either coloring $y_{1,1}$ with 1 yields a rainbow coloring or coloring $y_{1,1}$ with 2 does.

Case 4.3.4.2: Each of $y_{1,1}$ and $y_{1,2}$ has a neighbor of color 2 that has another neighbor of color 5. Since $y_{1,1}$ is not rainbow, the fourth neighbor of $y_{1,1}$ has color $c \in\{2,3,4\}$. Since $y_{1}$ cannot be recolored with 3 or 4 , some neighbor, say $r$, of $y_{1,1}$ of color $c$ has another neighbor of color 5 . If in the graph $G-y_{1} y_{1,1}, y_{1,1}$ can be recolored with 1 , then we may recolor $y_{1}$ with 3 and get a rainbow coloring of $G$. If $y_{1,1}$ can be recolored with either of 6 or 7 , then we have Case 4.3.3. To disallow coloring $y_{1,1}$ with 1,6 , and $7, r$ must be adjacent to vertices with each of these colors. By the symmetry between colors 3 and 4 , we assume that $f(r) \neq 4$. If the neighbor $r^{\prime}$ of $y_{1,1}$ with $f\left(r^{\prime}\right)=4$ has no neighbor of color $c^{\prime} \in\{6,7\}$, then we recolor $r$ with 4 and $y_{1,1}$ with $c^{\prime}$ thus getting Case 4.3.3. If $r^{\prime}$ has no neighbor of color 1 , then we recolor $r$ with $4, y_{1,1}$ with 1 , and $y_{1}$ with 3 obtaining a rainbow coloring. Finally if $f\left(N\left(r^{\prime}\right)-y_{1,1}\right)=\{1,5,6,7\}$, then we recolor $r^{\prime}$ with $3, y_{1,1}$ with 4 , and $y_{1}$ with 3 .

The last subcase is:

Case 4.3.4.3: $y_{1,1}$ has no neighbor of color 2 that has another neighbor of color 5 . Then recoloring $y_{1}$ with 2 creates another admissible coloring $f^{\prime}$. We may then repeat our previous argument with $y_{3}$ playing the role of $y_{2}$ to conclude that $y_{3}$ has neighbors of color 6 and 7. If $y_{3}$ is admissible, then repeating the above argument we conclude that $y_{3}$ may be recolored with color 1 in the original coloring $f$. Then after this recoloring, by coloring $x$ with 2 we get a rainbow coloring. Also, if $y_{2}$ is admissible in $f$, then we may recolor both of $y_{1}$ and $y_{2}$ with 2 and color $x$ with 1 to get a rainbow coloring. Hence we may assume that all the neighbors of $y_{2}$ and $y_{3}$ apart from $x$ are colored with 6 or 7 . Recall that for $i=4,5$ and $j=1,2,3, f\left(y_{i, j}\right)=j+2$ and $y_{i, 4}$ is uncolored. If for some $i \in\{4,5\}, y_{i, 4}$ has no neighbor of color $c \in\{6,7\}$, then we can color $y_{i}$ with $c$ and get a better admissible coloring. Since none of $y_{1}, y_{2}$, or $y_{3}$ has a neighbor with color 3 , if $y_{4,4}$ has no neighbor of color 1 or $y_{5,4}$ has no neighbor of color 2 , then by coloring $y_{4}$ with $1, y_{5}$ with 2 and $x$ with 3 creates an admissible coloring with more colored vertices. By the symmetry between colors 1 and 2, each of $y_{4,4}$ and $y_{5,4}$ has neighbors of colors $1,2,6$, and 7 .

If $y_{4,1}$ does not have a neighbor of color $c^{\prime} \in\{1,2,6,7\}$, then coloring $y_{4,4}$ with $3, y_{4}$ 
with $c^{\prime}$ and $x$ with 4 yields an admissible coloring. Otherwise, we recolor $y_{4,1}$ with 4 and color $y_{4}$ with 3 . This proves the lemma.

\section{Acknowledgment}

We thank the referees for the helpful comments.

\section{References}

[1] M. O. Albertson and D. M. Berman, The acyclic chromatic number, Congressus Numerantium, No. XVII (1976), Utilitas Math., Winnipeg, Man., 51-69.

[2] M. O. Albertson and D. M. Berman, Every planar graph has an acyclic 7-coloring, Israel J. Math. 28 (1977), 169-174.

[3] M. O. Albertson and D. M. Berman, An acyclic analogue to Heawood's theorem, Glasgow Math. J. 19 (1978), 163-166.

[4] M. O. Albertson, G. G. Chappell, H. A. Kierstead, A. Kündgen and R. Ramamurthi, Coloring with no 2-colored $P_{4}$ 's, Electron. J. Combin. 11 (2004), R\#26.

[5] N. Alon, C. McDiarmid and B. Reed, Acyclic coloring of graphs, Random Structures Algorithms 2 (1991), 277-288.

[6] M. I. Burstein, Every 4-valent graph has an acyclic 5-coloring, Soobšč. Akad. Nauk Gruzin. SSR 93 (1979), 21-24.

[7] G. Fertin and A. Raspaud, Acyclic coloring of graphs of maximum degree five: nine colors are enough, Information Processing Letters 105 (2008), 65-72.

[8] B. Grünbaum, Acyclic colorings of planar graphs, Israel J. Math 14 (1973), 390-408.

[9] H. Hocquard, M. Montassier, Acyclic coloring of graphs with maximum degree five, http: //hal.archives-ouvertes.fr/hal-00375166/en/.

[10] A. V. Kostochka, Upper bounds on the chromatic characteristics of graphs, PhD Thesis, Institute of Mathematics, Novosibirsk, 1978.

[11] K. Kothapalli, V. Satish and V. Ch. Venkaiah, Acyclic vertex coloring of graphs of maximum degree $\Delta$, Talk at 75 th Annual conference of the Indian Math. Society, Kalasalingam Univ., Krishnakoil, December 2009.

[12] K. Kothapalli, S. Varagani, V. Ch. Venkaiah and K. Yadav, Acyclic Vertex Coloring of Graphs of Maximum Degree 5, Proc. Int. Conf. on Graph Theory and its Applications, Amrita Vishwa Vidyapeetham, Coimbatore, December, 2008. 